\title{
Navigational strategy used to intercept fly balls under real-world conditions with moving visual background fields
}

\author{
Wei Wang • Michael K. McBeath • Thomas G. Sugar
}

Published online: 26 November 2014

(C) The Psychonomic Society, Inc. 2014

\begin{abstract}
This study explored the navigational strategy used to intercept fly balls in a real-world environment under conditions with moving visual background fields. Fielders ran across a gymnasium attempting to catch fly balls that varied in distance and direction. During each trial, the launched balls traveled in front of a moving background texture that was projected onto an entire wall of a gymnasium. The background texture consisted of a field of random dots that moved together, at a constant speed and direction that varied between trials. The fielder route deviation was defined as the signed area swept out between the actual running path and a straightline path to the destination, and these route deviation values were compared as a function of the background motion conditions. The findings confirmed that the moving visual background fields systematically altered the fielder running paths, which curved more forward and then to the side when the background gradient moved laterally with the ball, and curved more to the side and then forward when the background gradient moved opposite the ball. Fielder running paths deviated systematically, in a manner consistent with the use of a geometric optical control strategy that helps guide real-world perception-action tasks of interception, such as catching balls.
\end{abstract}

Keywords Navigational strategy · Interception · Baseball · Outfielder · Moving visual background $\cdot$ Real-world task

\section{W. Wang}

Department of Psychology and Institute for Mind and Biology,

The University of Chicago, Chicago, IL, USA

e-mail: wwang3@uchicago.edu

\section{K. McBeath $(\bowtie)$}

Department of Psychology,

Arizona State University, Tempe, AZ, USA

e-mail: Michael.McBeath@asu.edu

T. G. Sugar

The Polytechnic School, Ira A. Fulton Schools of Engineering, Arizona State University, Mesa, AZ, USA

\section{Introduction}

This research examined how moving background properties may affect real-world navigational judgments when intercepting moving targets. Of particular interest here was the interception of distant airborne objects such as projected balls, for which depth information can be quite sparse, so that control models are based primarily on the optical angles between the fielder and the target.

Optical angle control models for human catching behavior

During dynamic tasks like interception, fielders must control the direction of their movement with respect to the environment in order to reach the desired destination. This phenomenon of how fielders navigate to exactly the right spot at the right time to catch a ball is referred to as the "outfielder problem," and a family of optical angle control models reliably characterize fielder behavior.

Chapman (1968) proposed an optical strategy, later characterized as optical acceleration cancellation (OAC), of how baseball outfielders might approach and intercept fly balls. He noted that in cases in which fielders are stationary or run directly forward or backward toward the ball destination, the tangent of the vertical optical angle ( $\alpha$ in Fig. 1) increases at a constant rate. As is shown in the figure, the optical ball trajectory that fielders strive to achieve is geometrically equivalent to that of an imaginary elevator rising from home plate at a constant velocity and tilted back (see the dashed angled line in Fig. 1) by the amount that fielders run forward or backward (Babler \& Dannemiller, 1993; McLeod \& Dienes, 1993, 1996; McLeod, Reed, \& Dienes, 2003, 2006; Michaels \& Oudejans, 1992). Other research has further confirmed that fielders maintain OAC even in extreme cases, such as pop-ups, in which balls can travel along dramatically nonparabolic trajectories (McBeath, Nathan, Bahill, \& Baldwin, 2008), and when 


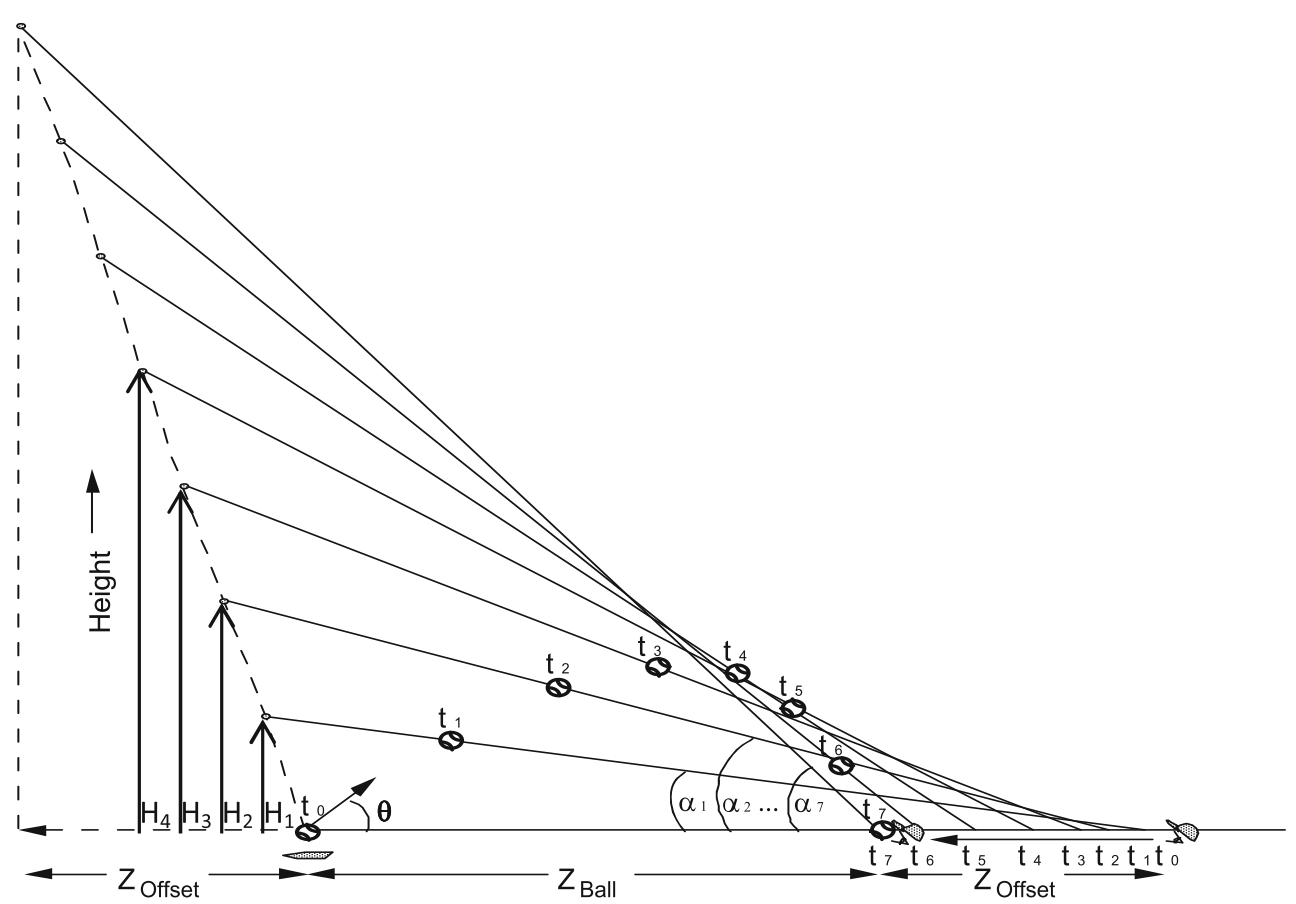

Fig. 1 Optical acceleration cancellation (OAC) model. A fielder runs along a path that keeps the tangent of the vertical optical ball angle $(\tan \alpha)$ increasing at a constant rate. Shown is a fielder approaching an airresistance-shortened ball trajectory, in equal temporal intervals $\left(t_{0}-t_{7}\right)$. $Z_{\text {Offset }}$ is the initial distance from the fielder to the ball destination point, and $Z_{\mathrm{Ball}}$ is the depth distance from the ball start to the destination point. The angled dashed line rising back from home plate illustrates the ends of the set of right triangles that increase linearly in height $(H)$ while maintaining the same depth distance to the fielder $\left(Z_{\text {Ball }}+Z_{\text {Offset }}\right)$; hence, $\tan \alpha$ $=H /\left(Z_{\text {Ball }}+Z_{\text {Offset }}\right)$ increases linearly over time catching fly balls in virtual environments (Fink, Foo, \& Warren, 2009; Turvey \& Fonseca, 2009; Zaal \& Bootsma, 2011).

McBeath, Shaffer, and Kaiser (1995a, 1995b) suggested another optical strategy, linear optical trajectory (LOT), which specifies that when fielders run to catch a ball, they move in such a way that the projected two-dimensional (2-D) optical trajectory of the ball remains linear while it monotonically increases. In other words, the 2-D projection of the three-dimensional (3-D) trajectory is divided into separate vertical and horizontal components, and fielders select an interception path that maintains a constant optical projection angle ( $\psi$ in Fig. 2), which is defined as the arccotangent (acot) of the rate of change in the horizontal optical angle to the target, $d \beta / \mathrm{d} t$, divided by the rate of change in the vertical optical angle, $\mathrm{d} \alpha / \mathrm{d} t$, resulting in an observed slope of $\psi=$ $\operatorname{acot}(d \beta / \mathrm{d} \alpha)$. From the viewpoint of the fielder, the angle between the observed horizontal level and the ball's instantaneous observed direction of movement is kept invariant by the fielder's choice of running path (Shaffer, Krauchunas, Eddy, \& McBeath, 2004; Shaffer \& McBeath, 2002; Shaffer, McBeath, Krauchunas, \& Sugar, 2008; Shaffer, McBeath, Roy, \& Krauchunas, 2003; Sugar, McBeath, \& Wang, 2006). The LOT can be viewed as a more general extension of the OAC, since for both strategies the fielder moves to null out acceleration, but for the OAC the nulling is only in the vertical direction, whereas for the LOT it is in both vertical and horizontal directions. This allows the LOT to handle balls hit off to the side, whereas the $\mathrm{OAC}$ requires specification of an additional lateral interception strategy. There has been some disagreement regarding the extent to which fielders maintain LOT during catching tasks within unnatural environments, such as virtual-reality settings that incorporate discretely changing trajectories (Fink et al., 2009; Turvey \& Fonseca, 2009), but such manipulations have been difficult to achieve in real-world settings. Other interception research in real-world environments has supported use of LOT when catching footballs (Shaffer, Dolgov, Maynor, \& Reed, 2013). In addition, Shaffer, Marken, Dolgov, and Maynor (2013) found that for fielders chasing toy helicopters flying along complex trajectories, pursuit behavior remained very well accounted for by a segmented LOT strategy (SLOT) in which the LOT parameters were reset by the fielder following dramatic changes in the target trajectory.

\section{Environmental influence on perception-action tasks}

Complex visual information needs to be integrated in order to successfully guide self-movement through real-world environments (Brouwer, Lopez-Moliner, Brenner, \& Smeets, 2006; Gigerenzer \& Goldstein, 2011; Marken, 2014). While walking down a busy street or crossing an intersection, the presence of moving objects, such as cars or pedestrians, might add potentially conflicting motion information to the scene that could cause biases in the control of heading. Warren and Saunders (1995) and Royden and Hildreth (1996) found that 
(a)

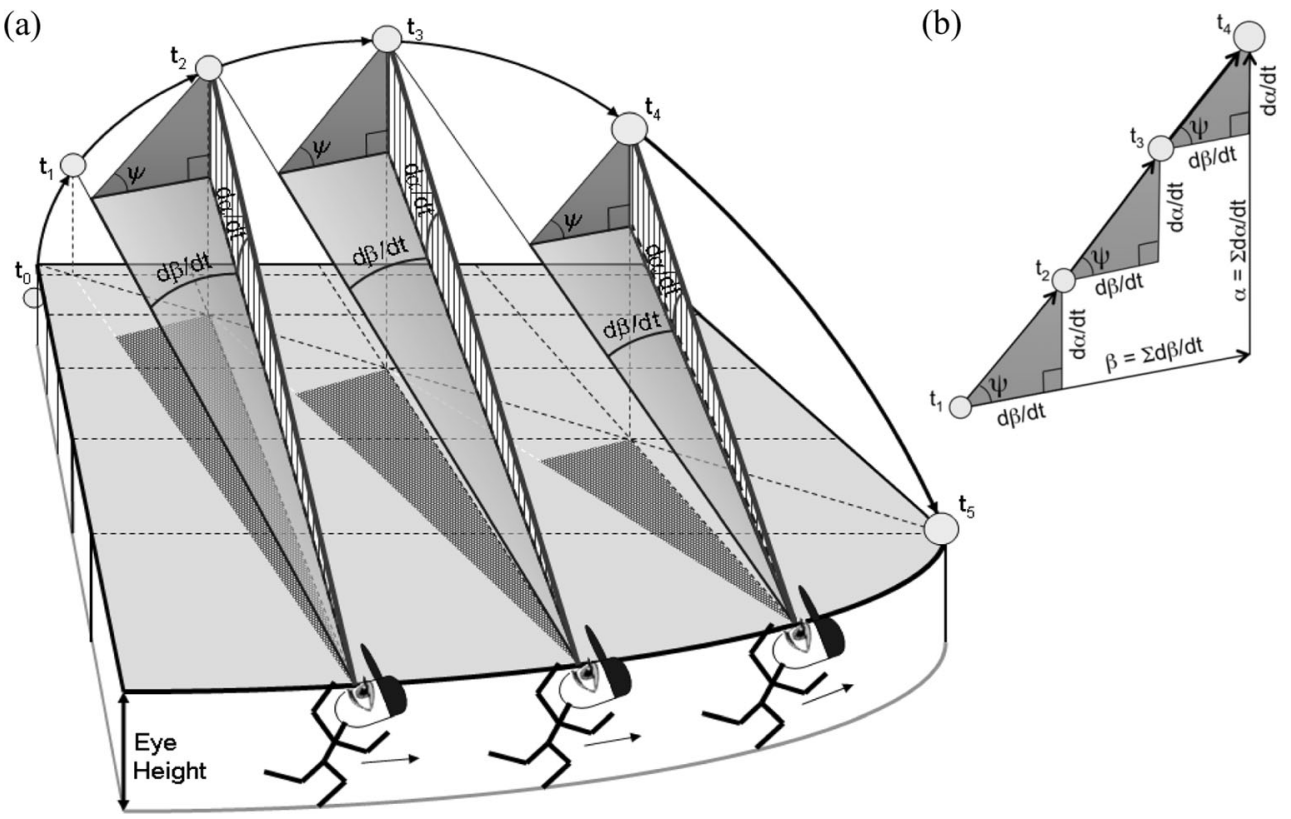

Fig. 2 Linear optical trajectory (LOT) model. When a fielder runs to the side, the observed relative position of the ball sweeps laterally and upward from its former position. The magnitude of the lateral optical change in the target position relative to the background over small periods of time can be designated as $d \beta / \mathrm{d} t$, and the lateral optical angle, $\beta$, equals $\int d \beta / \mathrm{d} t$. A LOT is achieved when the rate of change of $\beta$ is matched to the rate of change in the vertical optical angle, $\alpha, \operatorname{or} \operatorname{acot}(d \beta d \alpha)=$ constant $=$ $\psi$. Geometrically, the fielder moves such that the instantaneous triangular optical projections remain congruent over time. a 3-D diagram showing fielder maintaining the same projection angle, $\psi$, over several intervals of time. b 2-D projection of the optical ball trajectory from the fielder's perspective, with the projection triangles from panel (a) being tiled to form a continuous straight optical trajectory that is observed by the moving fielder in general the presence of a moving object does not significantly affect human heading judgments. However, when the object crosses the path, it causes small biases, and the direction of these biases depends on the position and direction of the moving object.

In baseball interception research, Oberle, Hollums, McBeath, and Terry (2006) examined the influence of irrelevant moving reference frames on estimating ball destination. In these experiments, balls were rolled from home plate toward random locations along a hidden barrier between first and second base, and confederate base runners and fielders served as distractors. The results showed that estimates of ball destination by stationary observers were significantly biased in the direction opposite to the confederates' motion, but estimates remained accurate for observers running toward the ball as fielders do. These findings confirm that in this real-world interception scenario, moving observers are relatively immune to distraction from background motion when engaged in interceptive perception-action activity. Stone, Dolgov, DaSilva, and McBeath (2008) tested whether background stimuli can impede the shooting accuracy of basketball free-throw shooting. In these experiments, images were projected onto a basketball backboard, either provocative pictures or continuous motion gradients at a variety of speeds, directions, and texture types. The results showed no general systematic effect on shooting accuracy due to background stimulus conditions, and in particular, background motion had essentially no effect. These findings provide further support for the robustness and independence of the perceptionaction system to resist distortion and distraction from background motion.

Oberle, Reagan, and Doyen (2006) compared novice and experienced basketball players on susceptibility to distraction by other players during shot attempts. The results confirmed a significantly higher percentage of made shots in the control condition (no defender) than in the experimental condition (defender tried to block the shot from the front). The findings verified that some kinds of background motion can reduce perception-action performance - perhaps ones that are highly embedded with the action task being performed. Additionally, several studies have shown that observers generally experience an axis-aligned motion (AAM) bias (a misjudgment of the destination of a moving object that travels along a trajectory misaligned with its own axis; Dolgov, McBeath, \& Sugar, 2009; Morikawa, 1999). Dolgov, Birchfield, McBeath, Thornburg, and Todd (2009) had both moving and stationary observers judge the final destinations of floor-projected moving geometric shapes in a large, immersive mixed-reality environment. The results showed that when observers were allowed to move and engage the action system, judgments were significantly more accurate than when the observers were stationary. The results are also consistent with neural pathway models which specify 
that illusions are diminished when observers take part in perception-action tasks (Goodale \& Milner, 1992; Milner \& Goodale, 2008).

\section{Purpose of the present research}

Some research supports the idea that background movement should have little impact on the chosen interceptive route during navigational pursuit of a projectile target (Oberle, Hollums, et al. 2006; Royden \& Hildreth, 1996; Stone et al., 2008; Warren \& Saunders, 1995). In contrast, other work supports the idea that background movement should systematically alter the chosen interceptive route (Dolgov, Birchfield, et al. 2009; Oberle, Reagan, et al. 2006). In addition, much of the past research has used computer monitors or small virtual environments to simulate flow fields (Royden \& Hildreth, 1996; Warren \& Saunders, 1995). However, the wide angle and interactive characteristics of flow fields suggests that providing observers with a large, immersive environment of flow fields might increase the impact of the distracting background motion. Recent interception research in larger virtual environments has addressed some of these issues, but still it has been limited by display resolution and reaction speed and the lack of accommodation depth cues, all of which prevent precise, rapid actions like catching from being fully realistic (Fink et al., 2009; Turvey \& Fonseca, 2009; Zaal \& Bootsma, 2011). In short, it remains unclear whether or not moving background stimuli affects human catching behavior in a study under real-world conditions.

The present study tested whether moving background fields distorted fielder running paths while fielders were catching fly balls in a real-world environment. Continuous motion gradients (random-dot fields) were used to induce added systematic sensory background movement. The principal research question was to explore how optical control angles are determined. Specifically, does a fielder have some type of internal compass that only requires monitoring of the ongoing relative positions of him- or herself and the target, or is the background scenery used to calibrate the changes? In other words, in these types of interceptive action tasks, does some kind of direct perceptual coupling between the fielder and the target allow the background scenery to be ignored, or does the fielder use the background scenery to calibrate the optical angle to the target movement. If the former is true, then the moving backgrounds should have little effect on the running paths in the real-world interceptive perception-action tasks. If the latter is true, then the moving backgrounds should systematically bend the running paths in the direction opposing the background movement.

\section{Method}

Participants

Eleven volunteers from Arizona State University participated in the experiment. All had normal or corrected-to-normal vision, and were skilled in ball-catching sports. All were naïve to the purpose of the experiments, and completed consent forms in accordance with the policy of the Institutional Review Board.

\section{Apparatus}

The study was performed in a gymnasium at Arizona State University (length $25 \mathrm{~m} \times$ width $16 \mathrm{~m} \times$ height $7 \mathrm{~m}$ ). An eightcamera high-speed Vicon motion-capture system was used to record both the running path of the fielder and the trajectory of the fly ball. Three projectors, controlled in tandem by a single $\mathrm{PC}$, were used to project a moving visual background onto the entire back wall $(25 \times 5.2 \mathrm{~m})$ down to a height of $1.8 \mathrm{~m}$. The visual background consisted of random dots (each $0.57 \mathrm{deg}$ ), and the direction and speed of the dots was manipulated. Four spotlights were placed, one near each corner of the gymnasium, to provide light. A marker-covered helmet was used to indicate the ongoing position of the participant, and a markercovered ball $(13 \mathrm{~cm}$ in diameter) was used as the target to be caught (see Fig. 3).

\section{Procedure}

A fielder initially stood near the center of the gymnasium, facing the moving visual background wall. A thrower stood on the center of the long wall with the moving visual background, facing the fielder. For each trial, the thrower threw the ball so as to land near one of the four designated floor locations, and the fielder was unaware of the landing site and was instructed to catch the fly ball, irrespective of the moving visual background stimuli. The four locations were on either the left or right side in direction, and either far away ( 8 $\mathrm{m})$ or close $(4 \mathrm{~m})$ in distance, relative to the fielder.

The background dots could display four directions of movement (up left, up right, down left, and down right). The consistency of the direction of background movement relative to that of the ball was framed to best specify the hypotheses. Here consistent indicates that the background motion had the same lateral direction as the ball movement (both moving either left or right), and inconsistent indicates that the background motion was in the opposite lateral direction from the ball movement (see Fig. 4).

In addition, the background dots had two speeds (slow and fast). Here, slow indicates $2 \mathrm{~m} / \mathrm{s}$, a lateral speed that was typically slower than the initial lateral speed of the target ball; and fast indicates $8 \mathrm{~m} / \mathrm{s}$, a lateral speed that was typically faster 
(a)

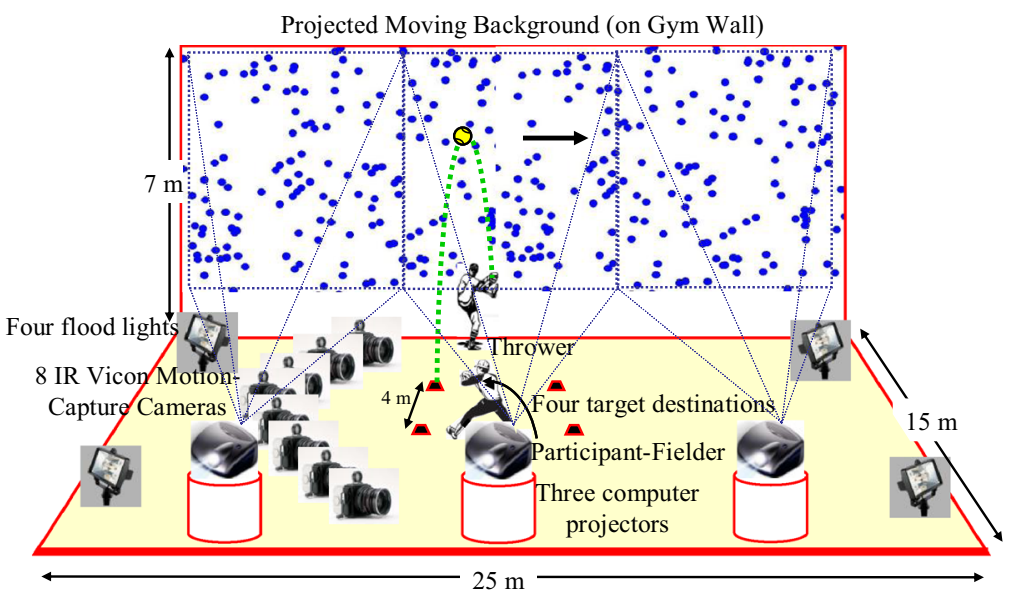

(b)

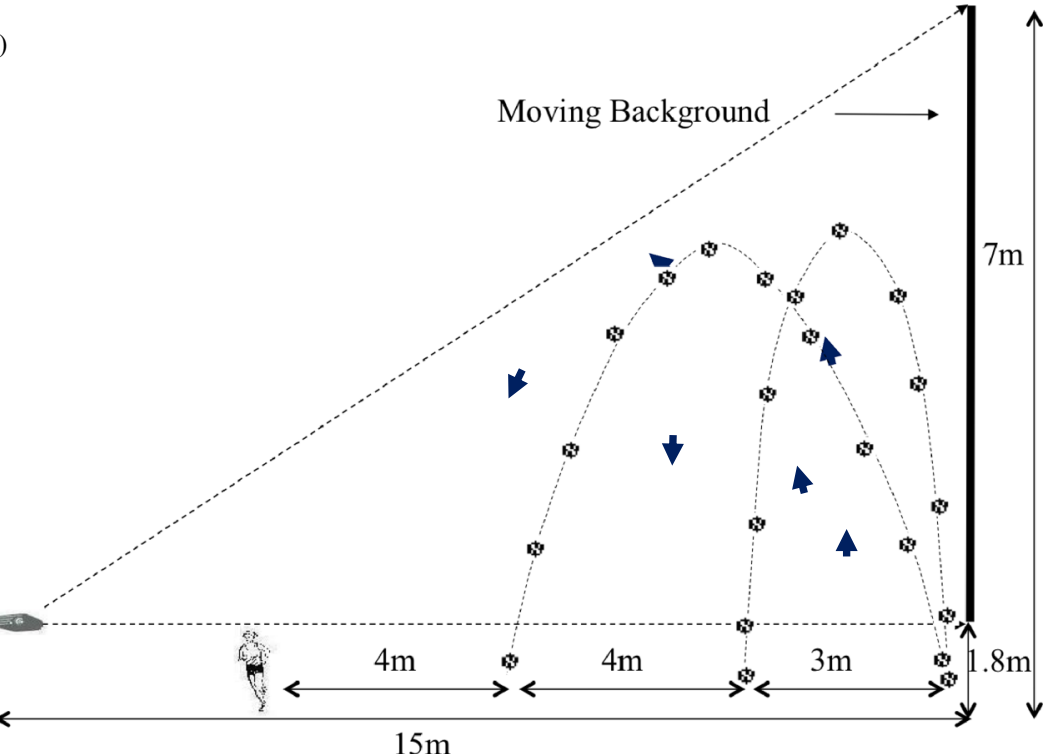

Fig. 3 View of the experimental setup. a 3-D view of the locations of the fielder, the thrower, the motion-capture cameras, the projectors, and the spotlights. The indicated four target destinations of the fly ball are known only to the thrower. $\mathbf{b}$ Side view of the height of the projections, the range

than the initial lateral speed of the target ball. The initial lateral speed of the target ball matched a background speed of about 4 $\mathrm{m} / \mathrm{s}$. Because the fielder also typically moved at several meters/ second in a diagonal direction toward the projection wall, the optical angular speed of the background dots varied widely over a range of the single digits $\left(0^{\circ}-9^{\circ} / \mathrm{s}\right)$ in the slow condition, and up to double digits $\left(20^{\circ}-30^{\circ} / \mathrm{s}\right)$ in the fast condition.

These resulted in 32 trials for each fielder. All of the trials were randomly sequenced. Each trial typically lasted about $2 \mathrm{~s}$.

For analyses, all running paths were rotated such that the initial-to-final running direction was set as the horizontal axis. The principal dependent variable was the running-path deviation, $\boldsymbol{D}$, defined as the signed area swept out between the actual running path and a straight-line route. Higher (positive, $\boldsymbol{D}>0$ ) signed deviation areas indicate that the fielder ran more to the side of the visual moving background on the projection wall, and sample fly ball trajectories for both close and far conditions. The $1.8-\mathrm{m}$ projection gap near the floor eliminated potential shadows from the thrower and the fielder

initially and accelerated more forward later on. Lower (negative, $\boldsymbol{D}<0$ ) signed deviation areas indicate that the fielder ran more forward initially and headed more to the side later on. In short, the magnitude of $\boldsymbol{D}$ indicates the extent of path curvature, and the sign indicates the direction of the curvature.

\section{Hypotheses}

1. The signed deviation area of the running paths, $\boldsymbol{D}$, systematically increases across the four consistency-byspeed conditions: consistent-fast, consistent-slow, inconsistent-slow, and inconsistent-fast. The logic of this prediction is that the optical projection angle ( $\psi$ in Fig. 5) initially decreases most in the inconsistent-fast condition 
(a)

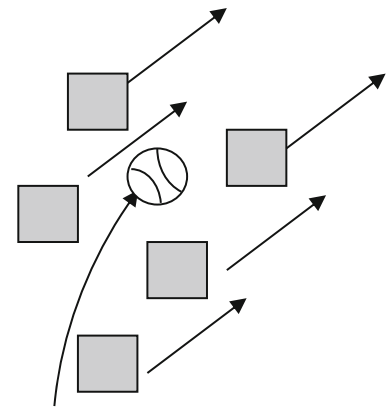

(c)

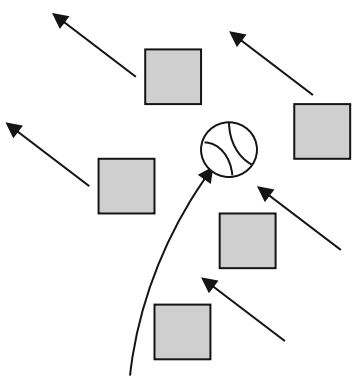

(b)

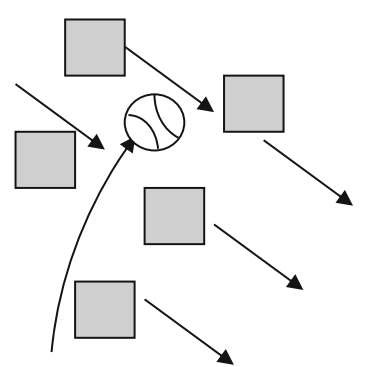

(d)

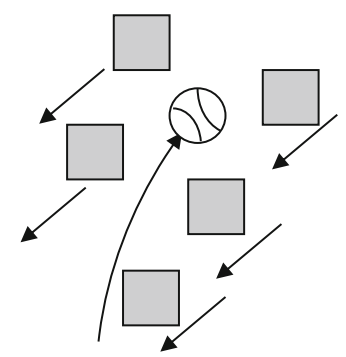

Fig. 4 Explanation of consistency (texture dots are represented as gray squares). The consistent condition occurs when both the target ball and the background texture move in the same lateral direction. This is shown here with the ball moving upward to the right and the texture moving a up-right and $\mathbf{b}$ down-right. The inconsistent condition occurs when the target ball moves in the opposite lateral direction from the background texture. This is shown here with the ball moving upward to the right and the texture moving $\mathbf{c}$ up-left or $\mathbf{d}$ down-left

and increases most (even crossing over $90^{\circ}$ ) in the consistent-fast condition. In order to keep $\psi$ constant to catch the fly ball, the fielder initially runs a greater distance to the side to increase $\psi$ and runs a lesser distance to the side to decrease $\psi$ (Figs. 5 and 6).

Generally, when a fielder initially runs a greater distance to the side, this reduces the relative lateral distance that the ball will need to travel, causing the ball's relative motion to angle more upward, hence increasing $\psi$. If the fielder runs far enough to the side, the fielder can reach a state in which the ball has no lateral movement and is headed straight up vertically, directly toward the fielder. As for the predicted paths, the fielder would initially run a greater distance forward to the fly ball in the consistentfast condition, initially run a lesser distance forward in the consistent-slow condition, initially run somewhat more of a distance to the side in the inconsistent-slow condition, and initially run the greatest distance to the side in the inconsistent-fast condition (Fig. 7). Statistically, the signed deviation area in these conditions is predicted to systematically differ and monotonically increase. $\boldsymbol{H}_{\mathbf{1}}: \boldsymbol{D}_{\mathrm{cf}}<\boldsymbol{D}_{\mathrm{cs}}<\boldsymbol{D}_{\text {is }}<\boldsymbol{D}_{\text {if. }}$

2. The signed deviation area of the running paths, $\boldsymbol{D}$, differs between the upward versus downward conditions of the moving background texture. The logic of this prediction is

that the optical projection angle, $\psi$, initially decreases in the upward condition relative to the downward condition. Thus, in the upward conditions, the fielder initially runs a greater distance to the side to increase $\psi$, and in the downward conditions initially runs relatively more forward toward the fly ball to decrease $\psi$ (Figs. 5 and 6). For the predicted running paths, the fielder initially runs a greater distance to the side in the upward condition and initially runs relatively more forward toward the fly ball in the downward condition. Statistically, the signed deviation area in the downward background conditions is predicted be lower than in the upward conditions. $\mathbf{H}_{\mathbf{2}}$ : $\boldsymbol{D}_{\text {downward }}<\boldsymbol{D}_{\text {upward }}$.

3. The signed deviation area of the running paths, $\boldsymbol{D}$, differs in the far and close conditions. The far condition is closer to the back projection wall, which could amplify its effect and generally increase the path deviation area. Alternatively, if the fielder exhibits a tendency to initially head toward the mean location of the two target distances used, this generally has the opposite effect, and decreases the curvature measure of the deviation area in the far condition. Statistically, the signed deviation area in the close depth distance condition is predicted to differ from the far depth distance condition. $\mathbf{H}_{\mathbf{3}}: \boldsymbol{D}_{\text {close }} \neq \boldsymbol{D}_{\text {far }}$.

4. There is no inherent geometric asymmetry between the half of the trials in which the fielder runs leftward versus rightward. Yet, there is a possibility that something like asymmetry of handedness in catching could affect the running paths, so both running directions were included, with the expectation of collapsing the results across the leftward versus the rightward conditions if the predicted null hypothesis is verified. Statistically, the signed deviation area of the running paths is predicted to not differ across lateral directions, either left or right, relative to the fielder. $\mathbf{H}_{4}: \boldsymbol{D}_{\text {left }}=\boldsymbol{D}_{\text {right }}$.

\section{Results}

The signed deviation area was initially analyzed using a $2 \times 2$ $\times 2 \times 2 \times 2$ analysis of variance (ANOVA), with 2 levels of consistency of the background and ball lateral directions (consistent vs. inconsistent) $\times 2$ background speeds (slow vs. fast) $\times 2$ background vertical directions (upward vs. downward) $\times 2$ target depth distances (close vs. far) $\times 2$ target lateral directions (left vs. right), where all five variables were manipulated within subjects. To more precisely explore the interaction between consistency and speed, the data were categorized into four groups (consistent-fast, consistent-slow, inconsistentslow, and inconsistent-fast), and a one-way ANOVA and planned polynomial contrasts were conducted across the four groups. 


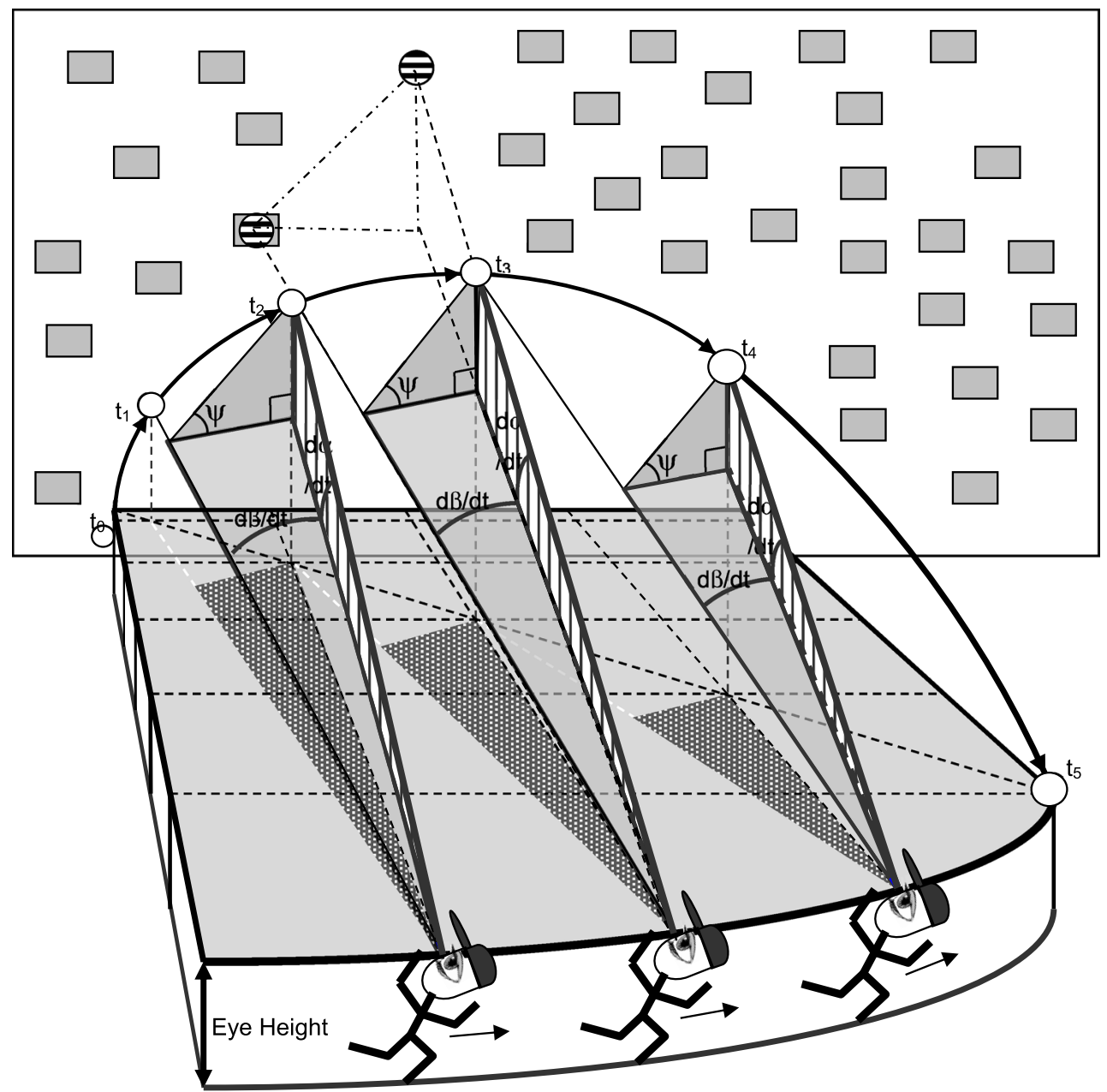

Fig. 5 Explanation of a hypothesis based on the linear optical trajectory heuristic. When a fielder runs to catch the ball, the moving background dots may distort the perception of the target ball trajectory relative to the back wall. The projection triangle for the middle fielder position is shown reprojected onto the back wall, with the striped circles indicating the two reprojected ball positions. The squares represent the background field spots at one instant in time, with one directly behind the previous location of the ball. Figure 6 shows a blow-up of the reprojected ball movement triangle in different conditions of background movement, with the gray squares indicating the second frame locations of the dot that was immediately behind the ball on the first frame (i.e., the striped circle on top of the gray square is the projected image of the ball at time $t_{2}$, and the striped circle on the white background is the projected image at time $t_{3}$ )
Following are the statistical results for each of the hypotheses:

1. Figure 8 illustrates a top view of running paths from typical trials in each of the four conditions: consistentfast, consistent-slow, inconsistent-slow, and inconsistentfast. The signed deviation areas differed significantly in the predicted monotonic order $[F(3,348)=12.38, p<.01$; Fig. 9a]. Polynomial contrasts verified that change in the means across four groups was strictly linear $[F(1,348)=$ $5.11, p<.05]$. This confirmed that running-path curvature exhibited a systematic pattern consistent with the predicted effect, due to laterally moving background direction and speed.

2. There was no main effect on running-path deviations due to the vertical direction of background motion [upward vs. downward: $F(1,10)=0.086, p=.775$ ] This supports the idea that vertical background flow has less impact on navigational route selection than does horizontal background flow.

3. The signed deviation area in the far condition exhibited significantly higher path curvature than it did in the close condition $[F(1,10)=84.05, p<.01$; Fig. 9b]. This confirmed that distance to the target has an influence on running-path curvature (Fig. 10).

4. The signed deviation area in the right condition was significantly higher than that in the left condition $[F(1$, $10)=5.43, p<.05$; Fig. 9c]. This confirmed that lateral running direction has an influence on running-path curvature. On average, targets to the right have destinations about $0.9 \mathrm{~m}$ more to the side than do ones to the left (Fig. 10). 


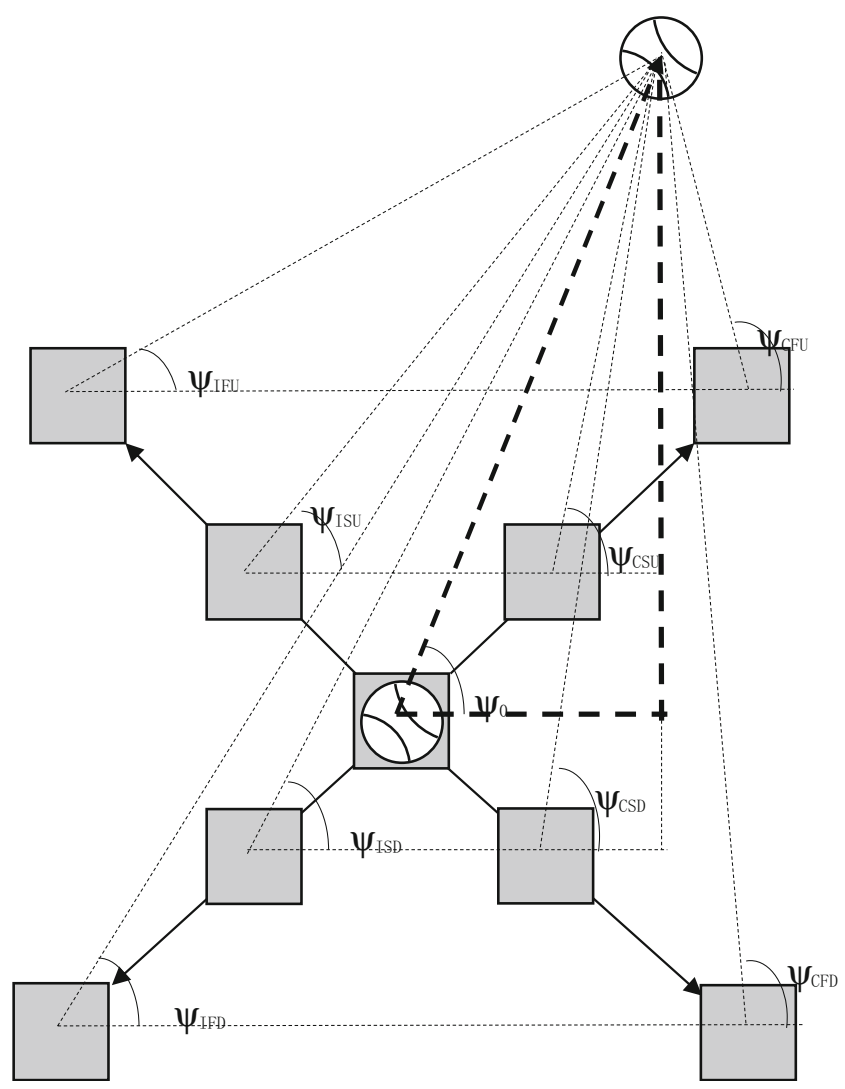

Fig. 6 Explanation of the consistency hypothesis. The triangle (solid dashes) is the same one shown between two frames in Fig. 5 for a rightward-moving ball. The square behind the initial location of the ball represents the location of the background field at time $t_{0}$, and the other eight squares indicate the observed locations of that same background dot in each of the eight background movement conditions at time $t_{1}$ (when the ball is now seen at the top of the triangle). The movement of the background dot (which serves as a reference point for the ball at $t_{0}$ ) will alter the experienced projection angle, $\psi$, in the following systematic ways. (i) When the image of the reference dot (square) moves left, the apparent $\psi$ angle decreases, and when the image of the reference dot (square) moves right, the apparent $\psi$ angle increases (and can actually cross to above $90^{\circ}$, in the extreme fast motion cases). (ii) When the image of the reference dot (square) moves laterally a given distance and moves up, the apparent $\psi$ angle is smaller than when it moves the same lateral distance, but down. (iii) In general, the changes are more dramatic in the fast-moving background conditions (depicted by the four outermost dots [gray squares]), and less dramatic in the slow-moving background conditions (depicted by the four innermost dots [gray squares])

Overall, the predicted model variables of the four hypotheses accounted for $77.1 \%$ of the variance in fielder runningpath deviation.

\section{Discussion}

This study confirmed that background movement properties affect real-world fielder running behavior in a predictable, systematic way across four consistency-by-speed conditions: consistent-fast, consistent-slow, inconsistent-slow, and inconsistent-fast. Specifically, fielders initially ran forward to the fly
Ball movement direction

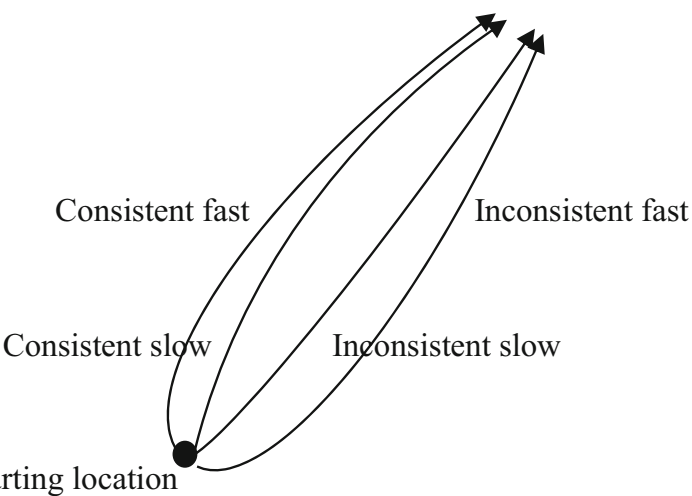

Fielder starting location

Fig. 7 Top view of the four predicted rightward running paths for the consistency-by-speed conditions (described in order of left to right). The fielder is predicted to initially run along the most forward-headed path in the consistent-fast condition, somewhat more to the side in the consistentslow condition, then even more to the side in the inconsistent-slow condition, and the most to the side in the inconsistent-fast condition. Mathematically, the running-path deviation, $\boldsymbol{D}$, will monotonically increase from the most negative (leftmost curvature) to the most positive (rightmost curvature)

ball the most in the consistent-fast condition, somewhat more to the side in the consistent-slow condition, even more to the side in the inconsistent-slow condition, and the most to the side in the inconsistent-fast condition. On average, there was a general tendency toward running paths with positive curvatures (i.e., cases in which the fielder initially headed more to the side, and later headed more forward), which had also been found in previous research on catching fly balls and pop-ups without background motion (McBeath et al., 1995a; McLeod, Reed, \& Dienes, 2001; Shaffer et al., 2008). In addition, this general running path deviation is consistent with control data that we collected in this setting, but with a stationary background.

One reason that we found little effect of the vertical component of background motion may have been that the vertical optical speed of the ball was considerably higher than its lateral optical speed. This resulted in the vertical background movement possibly not being fast enough to be in the range in which it could produce a notable interaction with the perception of optical ball speed. Additionally, the lack of statistical significance might reflect a small effect size.

Depth of running distance systematically affected fielder running paths, with fielders initially curving more forward toward the far fly ball, and relatively less so for the close fly ball. This finding was consistent with a "regression to the mean" tendency in the initial running direction, in which the fielder appeared to be initially biased to run toward the centroid of the two depths that were repeatedly presented, and then to later adjust the heading by running either more sideways for the close depth destination, or more forward for the far depth destination. 
(a) Consistent-fast

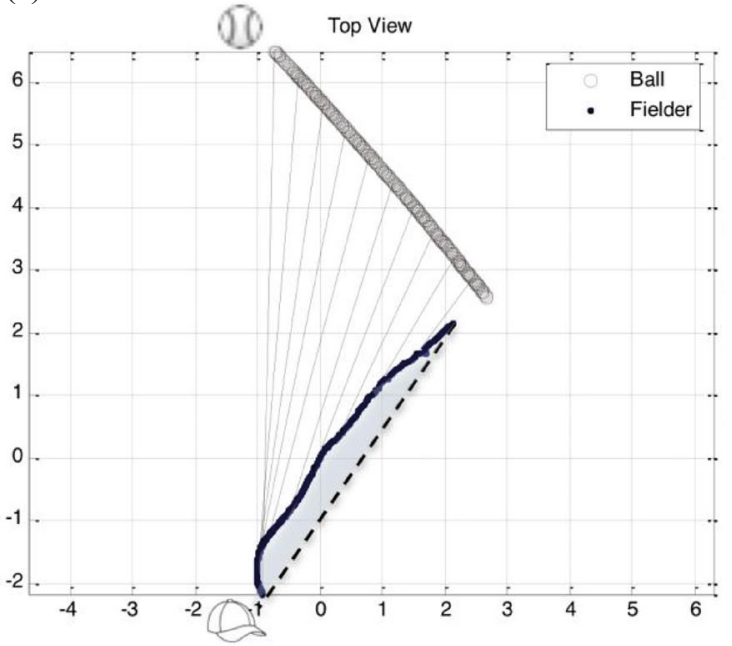

(c) Inconsistent-slow

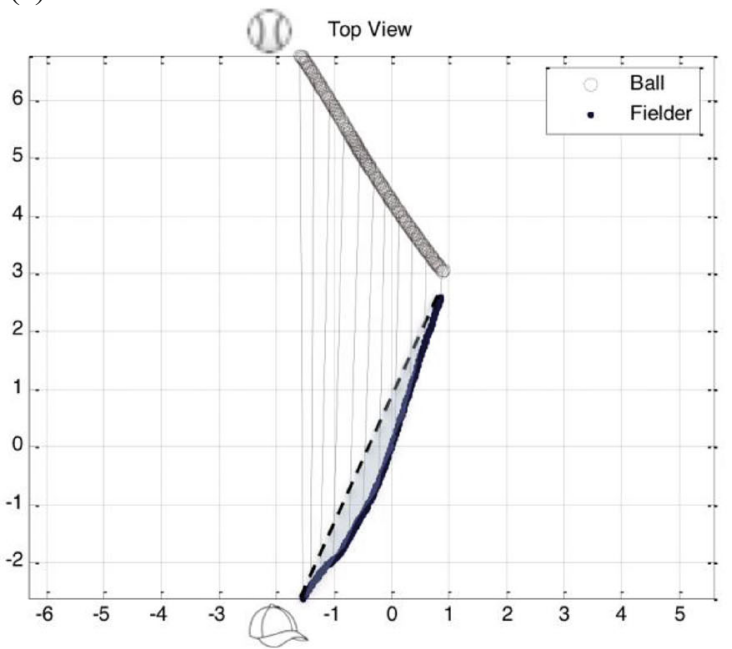

Fig. 8 Top view of four typical running paths to far-right destination. Alignment lines are shown in gray, and the initial positions of fielder and ball are indicated by hat and ball icons. The dotted diagonal lines specify the direct line-to-destination reference orientation by which running path curvature was calculated. Deviation, $\boldsymbol{D}$, is defined as the shaded area between the direct and actual running paths. a In the consistent-fast condition, a fielder initially runs more forward toward the fly ball, and the alignment lines progressively tilt more and more to the side. $\mathbf{b}$ In the

The lateral left versus right ball destination directions were found to affect the fielder running path curvature, with initial movement found to be more forward when running leftward and more to the side when running rightward. A likely reason for this unpredicted difference was an unplanned lateral shift in the centroids of the distributions of the leftward versus rightward target ball destinations. The asymmetric distribution of the target ball destinations ended up being $0.9 \mathrm{~m}$ farther from the thrower-fielder center axis on the right-side throws than on the left-side ones, possibly related to the thrower being righthanded. In addition, the lateral offset was consistent with a possible tendency for fielders to catch the ball with their (b) Consistent-slow

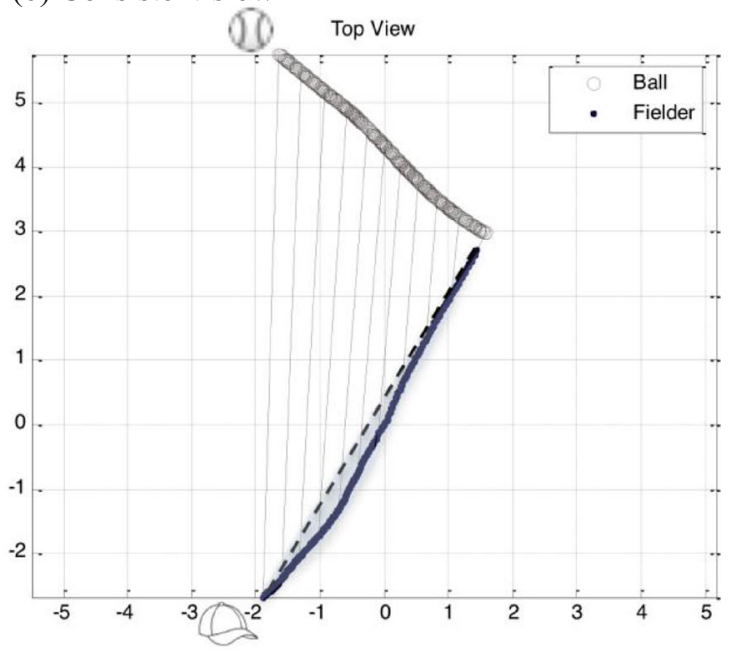

(d) Inconsistent-fast

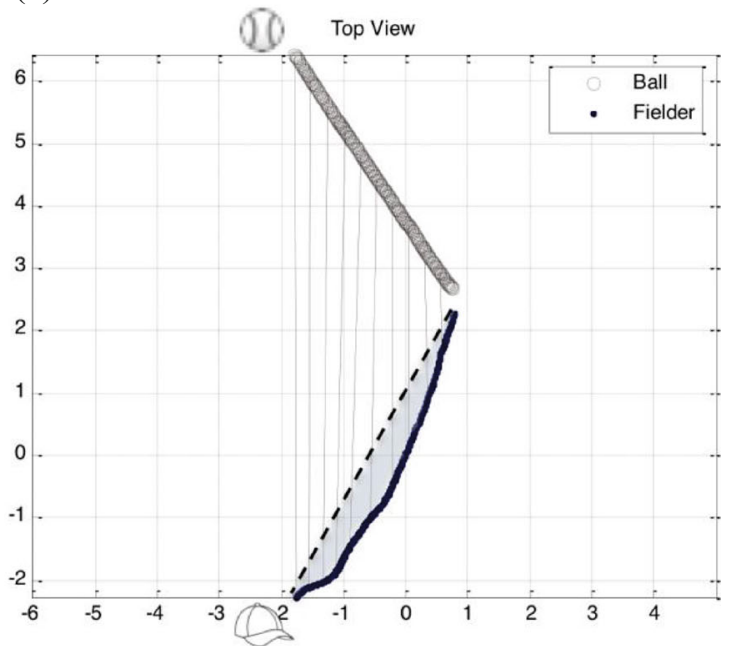

consistent-slow condition, a fielder initially runs somewhat to the side, and the alignment lines are closer to parallel. $\mathbf{c}$ In the inconsistent-slow condition, a fielder initially runs more to the side, and the alignment lines remain very close to parallel. $\mathbf{d}$ In the inconsistent-fast condition, a fielder initially runs largely to the side and even ahead of the ball such that the alignment lines start to tilt back beyond remaining parallel. The overall average deviations in these conditions are shown in Fig. 9a

left hand, as most right-handers are taught to do when wearing a baseball mitt.

\section{Catching strategy}

The present results are consistent with the use of an optical control strategy (such as LOT or a generic angular constancy metric) to guide real-world perception-action tasks of interception (Marken, 2014; McBeath et al., 1995a, 1995b; Shaffer, Dolgov, et al. 2013; Shaffer et al., 2004; Shaffer et al., 2008; Sugar et al., 2006). The fielder clearly appears to be using the background texture as a reference frame with which to compare successive optical ball positions, and any 

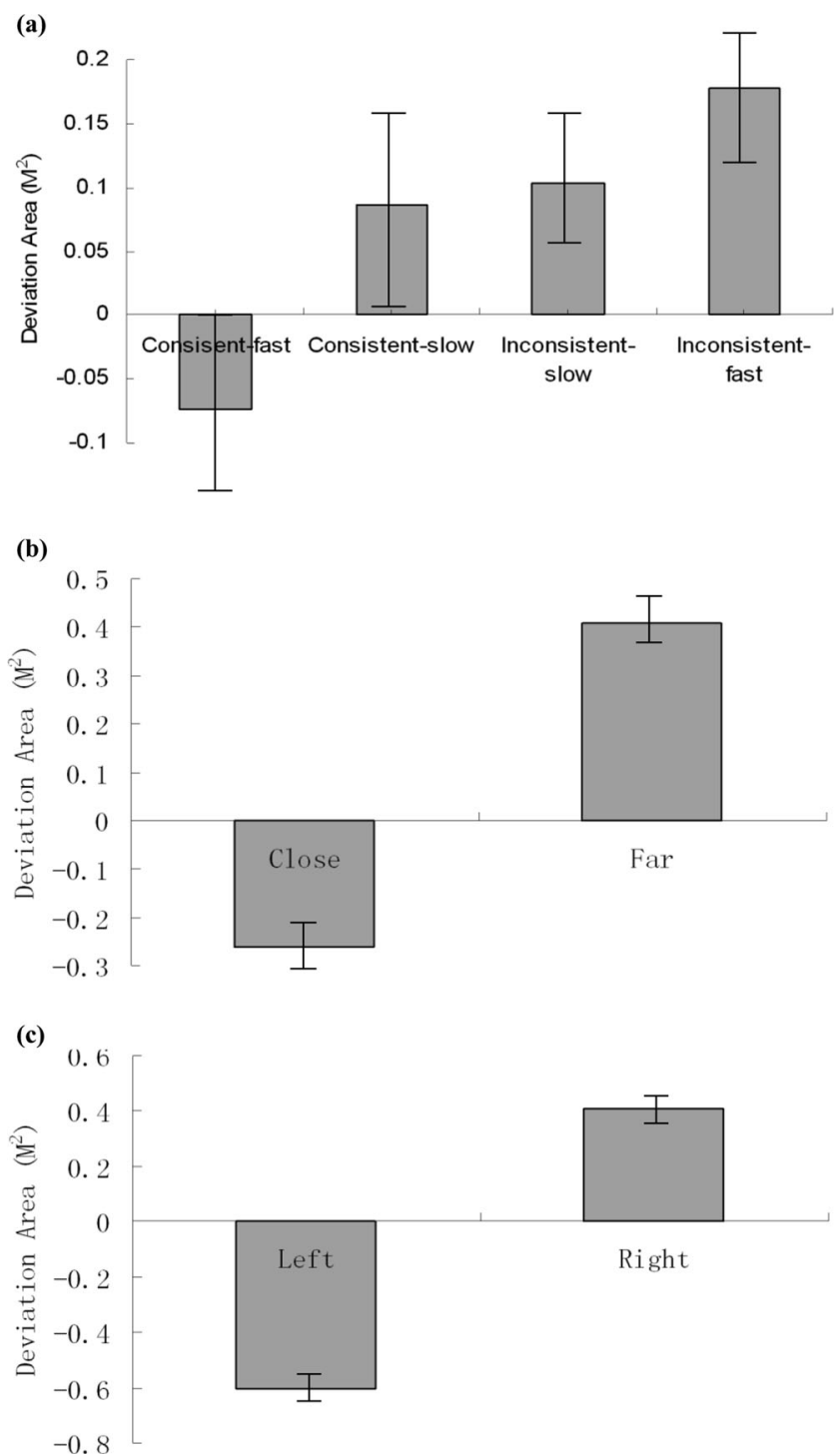

Fig. 9 Results due to a consistency and speed of background motion, b depth distance of the target ball, and $\mathbf{c}$ lateral direction of the target ball. Error bars indicate $\pm 1 S E$. Curvature is specified by the deviation area, $\boldsymbol{D}$, defined as the signed area swept out between the actual running path and a straight-line route (in meters squared). A positive $\boldsymbol{D}$ indicates that the fielder runs more to the side initially, whereas a negative $\boldsymbol{D}$ indicates that the fielder runs more forward initially

motion distortion in the background scenery can change the relative relationship between the image of the ball and the background reference frame. Therefore, fielders were systematically affected by the background texture motion when judging the ball location and selecting where to run. The findings do not support the idea that the fielder has some type of internalized position-monitoring system for judging orientation and maintaining lateral alignment with the target, independent of the background, nor that running-path curvature is due simply to some kind of inertial constraints, independent of the background. In short, the findings are not consistent with a self-contained direct-perception theory, in which the ongoing relative position of the target ball can accurately be determined

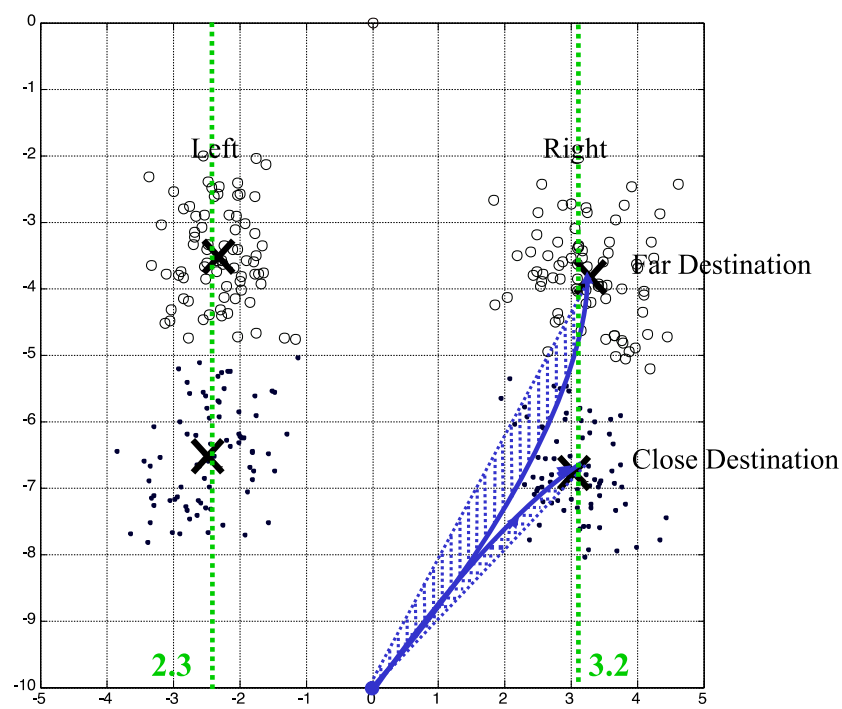

Fielder Start Point

Fig. 10 Top view of the fly ball destination distribution. The four conditions of the destination of the fly ball are close left, close right, far left, and far right (relative to the fielder). The landing locations of all of the far-destination trials are indicated by circles, whereas all of the closedestination trials are indicated by dots. Balls headed to the right landed on average about $0.9 \mathrm{~m}$ farther to the side, possibly related to the thrower being right-handed. Also shown are two typical running paths to the right for far- and close-destination points, illustrating how initially running toward a regression-to-the-mean location ends up inducing more positive curvature in the far condition

by utilizing perception-action dynamics solely based on the fielder-target geometry angles, independent of the background conditions. A component of optical control theory similar to maintaining the angular constancy of the target (such as a linear optical trajectory) appears to be necessary to explain fielder behavior.

In typical baseball interception tasks, a fly ball is hit or thrown near a fielder, and he or she develops a running path to catch it. One simple strategy for interception is to run directly toward the fly ball, effectively keeping at zero the lateral eccentricity angle between the direction of heading and the ball. This "pursuit" strategy produces an approach path that initially heads more forward and later more to the side, what we defined as negative curvature. Another strategy for the fielder is to laterally angle ahead of the ball, effectively maintaining a positive eccentricity angle that allows the fielder to remain laterally aligned with the fly ball. This "predictive" strategy typically leads to a path that is straighter and more direct than the pursuit path, and in an optimal case can even lead to a near straight-line approach path. Navigational research with animals has shown that their interception routes typically result in a combination between the pursuit and predictive paths (Fux \& Eilam, 2009; Kane \& Zamani, 2014; Olberg, 2012; Shaffer et al., 2004; Tucker, Tucker, Akers, \& Enderson, 2000). 
Since the background motion conditions varied from cases in which the ball moved with the background to cases in which it moved opposite the background, the interception strategies used by the fielders across the four conditions appear to be, respectively, biased toward either the pursuit strategy or the predictive strategy (Fig. 8). In the consistent-fast condition, the running path appears to be most similar to a pure pursuit path, in which the fielder continuously runs straight toward the target ball rather than trying to lead it, in effect acting the way that one would expect when the ball was headed right at him or her. Next, in the consistent-slow condition, the ongoing alignment lines are closer to parallel than are the ones in the consistent-fast condition. In the inconsistent-slow, and in particular the inconsistent-fast, conditions, the running paths appear to be more similar to a near-optimal predictive path, in which the fielder maintains nearly parallel alignment angles, and may even tend to run a bit ahead of the ball laterally. Thus, in addition to path curvature being consistent with the use of an optical control strategy, the alignment behavior also provides additional evidence in support of the use of background features to judge the ball movement direction. When a target object does not move laterally relative to the background, a pure pursuit strategy would normally aim directly toward the target, and when the ball does move laterally, more predictive weighting normally would provide a more direct path. Thus, it makes sense that when the background moves laterally with the ball, the fielder would be induced to initially run more directly toward the ball, as in a pursuit path. Our findings have verified that fielders behave in this manner, consistent with results from other species (Fux \& Eilam, 2009; Kane \& Zamani, 2014; Olberg, 2012; Shaffer et al., 2004; Tucker et al., 2000).

\section{Impact of background motion}

Goodale and Milner (1992; Milner \& Goodale, 2008) proposed separate visual pathways for action and perception in the visual system of the human brain. The dorsal stream, described as the "how" system, specifies action-relevant information, whereas the ventral stream, described as the "what" system, specifies perception-relevant information about objects. The present study supports the idea that a background movement effect is present even in these kinds of realworld perception-action tasks, in this case affecting the "how" system. This confirms that the control strategy guiding the perception-action "how" system relies on continuously monitoring visual background information, even in real-world interception tasks with background motion.

The findings of Stone et al. (2008) showed that moving background visual stimuli generally have no significant effect to impede the accuracy of basketball free-throw shooting. But in the present situation, the fielder moved in an action task with attention clearly focused on the target ball, and a moving background texture consisting of random dots systematically altered the fielder running paths. One possible explanation regarding these potentially conflicting findings could be the difference in tasks between stationary aiming and shooting and dynamic running. When the player performs free-throw shooting, he or she stands still, and the target basketball hoop is also stationary. Although moving background stimuli can vary in speed, direction, and texture type, the perceptionaction system appears to be able to resist distortion due to the background idiosyncrasies for such tasks. When the fielder runs to catch a target ball that is flying across the visual field, both the target and background are dynamically changing. In this active-interceptive situation, the moving background stimuli can systematically alter the perceived locations of the fly ball, and the perceptionaction system appears to need to use the background to calibrate position, leaving the system apparently prone to distortion. Thus, running to intercept a moving target in front of a moving background appears to be a task in which the dorsal (or "how" system) can be temporarily misled, though the fielder still usually converges to a successful interception location. Because the behavior of the "how" system is based on continuous updating of perception-action feedback loops, even initial systematic offsets are gradually corrected over time and merely result in a less-than-optimal route of convergence to the target. In the present study, the initial running misdirection (due to use of the moving background as a reference) leads the fielder to pursue the target from an angle somewhat offset from the optimal, straight-line running path.

Past research has documented other types of systematic interaction between the perception of moving foreground and background stimuli that may be influential in the present situation. For example, in the phenomenon of "motion repulsion" and the related phenomenon of "direction repulsion," a moving background can bias the direction of a transparent or simultaneously moving foreground by as much as $7^{\circ}$ (Grunewald, 2004; Levinson \& Sekuler, 1976; Rauber \& Treue, 1999; Treue, Hol, \& Rauber, 2000; Wiese \& Wenderoth, 2007). It is possible that as our fielders move closer to the ball and the background, the changes in the relative speed and direction of the background motion may produce a motion repulsion or direction repulsion effect that could in turn change the perceived optical angle of the ball and bias the running path. Yet, because the moving background is always present and the fielder motion is misdirected from the start, the motion repulsion effect does not appear to be the principal factor producing the nonoptimal running-path curvature. In the present case, fielder maintenance of optical angular constancy between 
the target and the background motion appears to be a more parsimonious explanation of why the fielder alters his or her running path.

Much of the past research on interceptive behavior with background movement has used either computer monitor displays or small virtual environments to simulate background flow fields (Royden \& Hildreth, 1996; Warren \& Saunders, 1995). Such settings typically lack active motion of the observer. Recent research has used largescale immersive virtual environments that have allowed fielder motion, but these can include distortion or some depth cue ambiguity, and they may limit the full speed and range of motion that can comprise accurate catching behavior (Fink et al., 2009; Turvey \& Fonseca, 2009; Zaal \& Bootsma, 2011). The present study is one of the first to investigate interceptive navigational behavior with added illusory flow fields in a real-world immersive environment. Our findings confirm that large-scale background motion can systematically influence real-time navigational strategies in real-world tasks in a manner that supports the concept that fielders use a dynamic angular optical control strategy such as maintaining a monotonically increasing linear optical trajectory (LOT).

\section{References}

Babler, T. G., \& Dannemiller, J. L. (1993). Role of image acceleration in judging landing location of free-falling projectiles. Journal of Experimental Psychology: Human Perception and Performance, 19(1), 15-31. doi:10.1037/0096-1523.19.1.15

Brouwer, A. M., Lopez-Moliner, J., Brenner, E., \& Smeets, J. B. J. (2006). Determining whether a ball will land behind or in front of you: Not just a combination of expansion and angular velocity. Vision Research, 46(3), 382-391.

Chapman, S. (1968). Catching a baseball. American Journal of Physics, $36(10), 868-870$.

Dolgov, I., Birchfield, D. A., McBeath, M. K., Thornburg, H., \& Todd, C. G. (2009a). Amelioration of axis-aligned motion bias for active versus stationary judgments of bilaterally symmetric moving shapes' final destinations. Attention, Perception, \& Psychophysics, 71(3), 523-529. doi:10.3758/APP.71.3.523

Dolgov, I., McBeath, M. K., \& Sugar, T. (2009b). Evidence for axisaligned motion bias: Football axis-trajectory misalignment causes systematic error in projected final destinations of thrown American footballs. Perception, 38(3), 399-410.

Fink, P. W., Foo, P. S., \& Warren, W. H. (2009). Catching fly balls in virtual reality: A critical test of the outfielder problem. Journal of Vision, 9(13), 14:1-14:8. doi:10.1167/9.13.14

Fux, M., \& Eilam, D. (2009). How barn owls (Typo alba) visually follow moving voles (Microtus socialis) before attacking them. Physiology and Behavior, 98(3), 359-366. doi:10.1016/j.physbeh.2009.06.016

Gigerenzer, G., \& Goldstein, D. G. (2011). The recognition heuristic: A decade of research. Judgment and Decision Making, 6(1), 100-121.

Goodale, M. A., \& Milner, A. D. (1992). Separate visual pathways for perception and action. Trends in Neurosciences, 15(1), 20-25. doi: 10.1016/0166-2236(92)90344-8
Grunewald, A. (2004). Motion repulsion is monocular. Vision Research, 44(10), 959-962.

Kane, S. A., \& Zamani, M. (2014). Falcons pursue prey using visual motion cues: New perspective from animal-born cameras. Journal of Experimental Biology, 217(2), 225-234.

Levinson, E., \& Sekuler, R. (1976). Adaptation alters perceived direction of motion. Vision Research, 16(7), 779-781.

Marken, R. S. (2014). Testing for controlled variables: A model-based approach to determining the perceptual basis of behavior. Attention, Perception, \& Psychophysics, 76(1), 255-263. doi:10.3758/s13414013-0552-8

McBeath, M. K., Nathan, A. M., Bahill, A. T., \& Baldwin, D. G. (2008). Paradoxical pop-ups: Why are they difficult to catch? American Journal of Physics, 76(8), 723-729.

McBeath, M. K., Shaffer, D. M., \& Kaiser, M. K. (1995a). How baseball outfielders determine where to run to catch fly balls. Science, 268(5210), $569-573$.

McBeath, M. K., Shaffer, D. M., \& Kaiser, M. K. (1995b). Play ballResponse. Science, 268(5218), 1683-1685.

McLeod, P., \& Dienes, Z. (1993). Running to catch the ball. Nature, 362(6415), 23.

McLeod, P., \& Dienes, Z. (1996). Do fielders know where to go to catch the ball or only how to get there? Journal of Experimental Psychology: Human Perception and Performance, 22(3), 531543. doi:10.1037/0096-1523.22.3.531

McLeod, P., Reed, N., \& Dienes, Z. (2001). Toward a unified fielder theory: What we do not yet know about how people run to catch a ball. Journal of Experimental Psychology: Human Perception and Performance, 27(6), 1347-1355. doi:10.1037/0096-1523.27.6.1347

McLeod, P., Reed, N., \& Dienes, Z. (2003). Psychophysics: How fielders arrive in time to catch the ball. Nature, 426(6964), 244-245.

McLeod, P., Reed, N., \& Dienes, Z. (2006). The generalized optic acceleration cancellation theory of catching. Journal of Experimental Psychology: Human Perception and Performance, 32(1), 139-148. doi:10.1037/0096-1523.32.1.139

Michaels, C. F., \& Oudejans, R. R. D. (1992). The optics and actions of catching fly balls: Zeroing out optical acceleration. Ecological Psychology, 4(4), 199-222.

Milner, A. D., \& Goodale, M. A. (2008). Two visual systems re-viewed. Neuropsychologia, 46(3), 774-785. doi:10.1016/j. neuropsychologia.2007.10.005

Morikawa, K. (1999). Symmetry and elongation of objects influence perceived direction of translational motion. Perception \& Psychophysics, 61(1), 134-143.

Oberle, C. D., Hollums, N. K., McBeath, M. K., \& Terry, D. P. (2006a). Motion by nearby players biases perception but not action in judgments of baseball destination. Perceptual and Motor Skills, 103(2), 585-606.

Oberle, C. D., Reagan, D. K., \& Doyen, J. (2006b). An ironic relationship between cognitive style and susceptibility to distraction in basketball. Journal of Sport and Exercise Psychology, 28, S141.

Olberg, R. M. (2012). Visual control of prey-capture flight in dragonflies. Current Opinion in Neurobiology, 22(2), 267-271. doi:10.1016/j. conb.2011.11.015

Rauber, H. J., \& Treue, S. (1999). Revisiting motion repulsion: Evidence for a general phenomenon? Vision Research, 39(19), 3187-3196.

Royden, C. S., \& Hildreth, E. C. (1996). Human heading judgments in the presence of moving objects. Perception \& Psychophysics, 58(6), 836856.

Shaffer, D. M., Dolgov, I., Maynor, A., \& Reed, C. (2013a). Receivers in American football use a constant optical projection plane angle to pursue and catch thrown footballs. Perception, 42(8), 813-827.

Shaffer, D. M., Krauchunas, S. M., Eddy, M., \& McBeath, M. K. (2004). How dogs navigate to catch frisbees. Psychological Science, 15(7), 437-441. 
Shaffer, D. M., Marken, R. S., Dolgov, I., \& Maynor, A. B. (2013b). Chasin' choppers: Using unpredictable trajectories to test theories of object interception. Attention, Perception, \& Psychophysics, 75(7), 1496-1506. doi:10.3758/s13414-013-0500-7

Shaffer, D. M., \& McBeath, M. K. (2002). Baseball outfielders maintain a linear optical trajectory when tracking uncatchable fly balls. Journal of Experimental Psychology: Human Perception and Performance, 28(2), 335-348. doi:10.1037/ 0096-1523.28.2.335

Shaffer, D. M., McBeath, M. K., Krauchunas, S. M., \& Sugar, T. G. (2008). Evidence for a generic interceptive strategy. Perception \& Psychophysics, 70(1), 145-157.

Shaffer, D. M., McBeath, M. K., Roy, W. L., \& Krauchunas, S. M. (2003). A linear optical trajectory informs the fielder where to run to the side to catch fly balls. Journal of Experimental Psychology: Human Perception and Performance, 29(6), 1244-1250. doi:10. 1037/0096-1523.29.6.1244

Stone, J., Dolgov, I., DaSilva, F., \& McBeath, M. (2008). Basketball free throw accuracy unaffected by projected background displays showing motion or emotion [Abstract]. Journal of Vision, 8(6), 1044. doi: $10.1167 / 8.6 .1044$
Sugar, T. G., McBeath, M. K., \& Wang, Z. (2006). A unified fielder theory for interception of moving objects either above or below the horizon. Psychonomic Bulletin \& Review, 13(5), 908-917.

Treue, S., Hol, K., \& Rauber, H. J. (2000). Seeing multiple directions of motion-physiology and psychophysics. Nature Neuroscience, 3(3), 270-276.

Tucker, V. A., Tucker, A. E., Akers, K., \& Enderson, J. H. (2000). Curved flight paths and sideways vision in peregrine falcons (Falco peregrinus). Journal of Experimental Biology, 203, 3755-3763.

Turvey, M. T., \& Fonseca, S. (2009). Nature of motor control: Perspectives and issues. Progress in Motor Control: Advances in Experimental Medicine and Biology, 629, 93-123. doi:10.1007/ 978-0-387-77064-2 6

Warren, W. H., \& Saunders, J. A. (1995). Perceiving heading in the presence of moving objects. Perception, 24(3), 315-331.

Wiese, M., \& Wenderoth, P. (2007). The different mechanisms of the motion direction illusion and aftereffect. Vision Research, 47(14), 1963-1967.

Zaal, F. T. J. M., \& Bootsma, R. J. (2011). Virtual reality as a tool for the study of perception-action: The case of running to catch fly balls. Presence, 20(1), 93-103. doi:10.1162/pres_a_00037 